\title{
On the entropy for group actions on the circle
}

by

\author{
Eduardo Jorquera (Santiago)
}

\begin{abstract}
We show that for a finitely generated group of $C^{2}$ circle diffeomorphisms, the entropy of the action equals the entropy of the restriction of the action to the nonwandering set.
\end{abstract}

1. Introduction. Let $(X$, dist) be a compact metric space and $G$ a group of homeomorphisms of $X$ generated by a finite family of elements $\Gamma=\left\{g_{1}, \ldots, g_{n}\right\}$. To simplify, we will always assume that $\Gamma$ is symmetric, that is, $g^{-1} \in \Gamma$ for every $g \in \Gamma$. For each $n \in \mathbb{N}$ we denote by $B_{\Gamma}(n)$ the ball of radius $n$ in $G$ (with respect to $\Gamma$ ), that is, the set of elements $f \in G$ which may be written in the form $f=g_{i_{m}} \cdots g_{i_{1}}$ for some $m \leq n$ and $g_{i_{j}} \in \Gamma$. For $g \in G$ we let $\|f\|=\|f\|_{\Gamma}=\min \left\{n: f \in B_{\Gamma}(n)\right\}$

As in the classical case, given $\varepsilon>0$ and $n \in \mathbb{N}$, two points $x, y$ in $X$ are said to be $(n, \varepsilon)$-separated if there exists $g \in B_{\Gamma}(n)$ such that dist $(g(x), g(y))$ $\geq \varepsilon$. A subset $A \subset X$ is $(n, \varepsilon)$-separated if all $x \neq y$ in $A$ are $(n, \varepsilon)$-separated. We denote by $s(n, \varepsilon)$ the maximal possible cardinality (perhaps infinite) of an $(n, \varepsilon)$-separated set. The topological entropy of the action at the scale $\varepsilon$ is defined by

$$
h_{\Gamma}(G \circlearrowright X, \varepsilon)=\limsup _{n \uparrow \infty} \frac{\log (s(n, \varepsilon))}{n},
$$

and the topological entropy is defined by

$$
h_{\Gamma}(G \circlearrowright X)=\lim _{\varepsilon \downarrow 0} h_{\Gamma}(G \circlearrowright X, \varepsilon) .
$$

Notice that, although $h_{\Gamma}(G \circlearrowright X, \varepsilon)$ depends on the system of generators, the properties of having zero, positive, or infinite entropy are independent of this choice.

The definition above was proposed in [5] as an extension of the classical topological entropy of single maps (the definition extends to pseudo-groups

2000 Mathematics Subject Classification: 20B27, 37A35, 37C85, 37E10.

Key words and phrases: topological entropy, group actions, circle diffeomorphisms. 
of homeomorphisms, and hence is suitable for applications in foliation theory). Indeed, for a homeomorphism $f$, the topological entropy of the action of $\mathbb{Z} \sim\langle f\rangle$ equals twice the (classical) topological entropy of $f$. Nevertheless, the functorial properties of this notion remain unclear. For example, the following fundamental question is open.

General Question. Is it true that $h_{\Gamma}(G \circlearrowright X)$ is equal to $h_{\Gamma}(G \circlearrowright \Omega)$ ?

Here $\Omega=\Omega(G \circlearrowright X)$ denotes the non-wandering set of the action, or in other words

$\Omega=\{x \in X:$ for every neighborhood $U$ of $x$, we have

$$
f(U) \cap U \neq \emptyset \text { for some } f \neq \text { id in } G\} .
$$

This is a closed invariant set whose complement $\Omega^{c}$ corresponds to the wandering set of the action.

The notion of topological entropy for group actions is quite appropriate in the case where $X$ is a one-dimensional manifold. In fact, in this case, the topological entropy is necessarily finite (cf. $\S 2)$. Moreover, in the case of actions by diffeomorphisms, the dichotomy $h_{\text {top }}=0$ or $h_{\text {top }}>0$ is well understood. Indeed, according to a result originally proved by Ghys, Langevin, and Walczak, for groups of $C^{2}$ diffeomorphisms [5], and extended by Hurder to groups of $C^{1}$ diffeomorphisms (see for instance [9]), we have $h_{\text {top }}>0$ if and only if there exists a resilient orbit for the action. This means that there exists a group element $f$ contracting an interval towards a fixed point $x_{0}$ inside, and another element $g$ which sends $x_{0}$ into its basin of contraction under $f$.

The results of this work give a positive answer to the General Question above in the context of group actions on one-dimensional manifolds under certain mild assumptions.

Theorem A. If $G$ is a finitely generated subgroup of $\operatorname{Diff}_{+}^{2}\left(\mathrm{~S}^{1}\right)$, then for every finite system of generators $\Gamma$ of $G$, we have

$$
h_{\Gamma}\left(G \circlearrowright \mathrm{S}^{1}\right)=h_{\Gamma}(G \circlearrowright \Omega) .
$$

Our proof for Theorem A actually works in the Denjoy class $C^{1+\text { bv }}$, and applies to general codimension-one foliations on compact manifolds. In the class $C^{1+\text { Lip }}$, it is quite possible that we could give an alternative proof using standard techniques from level theory $[2,6]$.

It is unclear whether Theorem A extends to actions of lower regularity. However, it still holds under certain algebraic hypotheses. In fact (quite unexpectedly), the regularity hypothesis is used to rule out the existence of elements $f \in G$ that fix some connected component of the wandering set and which are distorted, that is,

$$
\lim _{n \rightarrow \infty} \frac{\left\|f^{n}\right\|}{n}=0 .
$$


Actually, for the equality between the entropies it suffices to require that no elememt in $G$ be subexponentially distorted. In other words, it suffices to require that, for each element $f \in G$ with infinite order, there exist a non-decreasing function $q: \mathbb{N} \rightarrow \mathbb{N}$ (depending on $f$ ) with subexponential growth satisfying $q\left(\left\|f^{n}\right\|\right) \geq n$ for every $n \in \mathbb{N}$. This is an algebraic condition which is satisfied by many groups, for example nilpotent or free groups. (We refer the reader to [1] for a nice discussion of distorted elements.) Under this hypothesis, the following result holds.

TheOREM B. If $G$ is a finitely generated subgroup of $\mathrm{Homeo}_{+}\left(\mathrm{S}^{1}\right)$ without subexponentially distorted elements, then for every finite system of generators $\Gamma$ of $G$, we have

$$
h_{\Gamma}\left(G \circlearrowright \mathrm{S}^{1}\right)=h_{\Gamma}(G \circlearrowright \Omega) .
$$

The entropy of general group actions and distorted elements seem to be related in an interesting manner. Indeed, though the topological entropy of a single homeomorphism $f$ may be equal to zero, if this map appears as a subexponentially distorted element inside an acting group, then it may create positive entropy for the group action.

2. Some background. In this work we will consider the normalized length on the circle, and every homeomorphism will be orientation preserving.

We begin by noticing that if $G$ is a finitely generated group of circle homeomorphisms and $\Gamma$ is a finite generating system for $G$, then for all $n \in \mathbb{N}$ and all $\varepsilon>0$ one has

$$
s(n, \varepsilon) \leq \frac{1}{\varepsilon} \# B_{\Gamma}(n) .
$$

Indeed, let $A$ be an $(n, \varepsilon)$-separated set of cardinality $s(n, \varepsilon)$. Then for any two adjacent points $x, y$ in $A$ there exists $f \in B_{\Gamma}(n)$ such that $\operatorname{dist}(f(x), f(y))$ $\geq \varepsilon$. For a fixed $f$, the intervals $[f(x), f(y)]$ which appear have disjoint interiors. Since the total length of the circle is 1 , any given $f$ can be used in this construction at most $1 / \varepsilon$ times, which immediately gives (1).

Notice that, taking the logarithm on both sides of (1), dividing by $n$, and passing to the limit gives

$$
h_{\Gamma}\left(G \circlearrowright \mathrm{S}^{1}\right) \leq \operatorname{gr}_{\Gamma}(G),
$$

where $\operatorname{gr}_{\Gamma}(G)$ denotes the growth of $G$ with respect to $\Gamma$, that is,

$$
\operatorname{gr}_{\Gamma}(G)=\lim _{n \rightarrow \infty} \frac{\log \left(\# B_{\Gamma}(n)\right)}{n} .
$$

Some easy consequences of this fact are the following: 
- If $G$ has subexponential growth, that is, if $\operatorname{gr}_{\Gamma}(G)=0$ (in particular, if $G$ is nilpotent, or if $G$ is the Grigorchuk-Maki group considered in [8]), then $h_{\Gamma}\left(G \circlearrowright \mathrm{S}^{1}\right)=0$ for all finite generating systems $\Gamma$.

- In the general case, if $\# \Gamma=q \geq 1$, then from the relations

$\# B_{\Gamma}(n) \leq 1+\sum_{j=1}^{n} 2 q(2 q-1)^{j-1}= \begin{cases}1+\frac{q}{q-1}\left((2 q-1)^{n}-1\right), & q \geq 2, \\ 1+2 n, & q=1\end{cases}$

one concludes that

$$
h_{\Gamma}\left(G \circlearrowright \mathrm{S}^{1}\right) \leq \log (2 q-1) .
$$

This shows in particular that the entropy of the action of $G$ on $\mathrm{S}^{1}$ is finite. Notice that this may also be deduced from the probabilistic arguments of [3] (see Théorème D therein). However, these arguments only yield the weaker estimate $h_{\Gamma}\left(G \circlearrowright \mathrm{S}^{1}\right) \leq \log (2 q)$ when $\Gamma$ has cardinality $q$.

3. Some preparations for the proofs. The statements of our results are obvious when the non-wandering set of the action equals the whole circle. Hence, we will assume in what follows that $\Omega$ is a proper subset of $\mathrm{S}^{1}$, and we will denote by $I$ a connected component of the complement of $\Omega$. Let $\operatorname{St}(I)$ denote the stabilizer of $I$ in $G$.

Lemma 1. The stabilizer $\operatorname{St}(I)$ is either trivial or infinite cyclic.

Proof. The (restrictions to $I$ of the) non-trivial elements of $\left.\operatorname{St}(I)\right|_{I}$ have no fixed points, for otherwise these points would be non-wandering. Thus $\left.\operatorname{St}(I)\right|_{I}$ acts freely on $I$, and according to Hölder's Theorem $[4,7]$, its action is semiconjugate to an action by translations. We claim that if $\left.\operatorname{St}(I)\right|_{I}$ is nontrivial, then it is infinite cyclic. Indeed, if not then the corresponding group of translations is dense. This implies that the preimage by the semiconjugacy of any point whose preimage is a single point corresponds to a non-wandering point for the action. But this contradicts the fact that $I$ is contained in $\Omega^{c}$.

If $\left.\operatorname{St}(I)\right|_{I}$ is trivial then $\left.f\right|_{I}$ is trivial for every $f \in \operatorname{St}(I)$, and hence $f$ itself must be the identity. We then conclude that $\operatorname{St}(I)$ is trivial.

Analogously, $\operatorname{St}(I)$ is cyclic if $\left.\operatorname{St}(I)\right|_{I}$ is cyclic. In this case, $\left.\operatorname{St}(I)\right|_{I}$ is generated by the restriction to the interval $I$ of the generator of $\operatorname{St}(I)$.

Definition 1. A connected component $I$ of $\Omega^{c}$ will be called of type 1 if $\operatorname{St}(I)$ is trivial, and of type 2 if $\operatorname{St}(I)$ is infinite cyclic.

Notice that the families of connected components of type 1 and 2 are invariant, that is, for each $f \in G$ the interval $f(I)$ is of type 1 (resp. of type 2) if $I$ is of type 1 (resp. of type 2). Moreover, given two connected components of type 1 of $\Omega^{c}$, there exists at most one element in $G$ sending 
the former to the latter. Indeed, if $f(I)=g(I)$ then $g^{-1} f$ is in the stabilizer of $I$, and hence $f=g$ if $I$ is of type 1 .

Lemma 2. Let $x_{1}, \ldots, x_{m}$ be points contained in a single type 1 connected component of $\Omega^{c}$. If for some $\varepsilon>0$ the points $x_{i}, x_{j}$ are $(\varepsilon, n)$ separated for every $i \neq j$, then $m \leq 1+1 / \varepsilon$.

Proof. Let $I=] a, b\left[\right.$ be the connected component of type 1 of $\Omega^{c}$ containing the points $x_{1}, \ldots, x_{m}$. After renumbering the $x_{i}$ 's, we may assume that $a<x_{1}<\cdots<x_{m}<b$. For each $1 \leq i \leq m-1$ one can choose an element $g_{i} \in B_{\Gamma}(n)$ such that $\operatorname{dist}\left(g_{i}\left(x_{i}\right), g_{i}\left(x_{i+1}\right)\right) \geq \varepsilon$. Now, since $I$ is of type 1 , the intervals $] g_{i}\left(x_{i}\right), g_{i}\left(x_{i+1}\right)$ [ are pairwise disjoint. Therefore, the number of these intervals times their minimal length is less than or equal to 1 . This gives $(m-1) \varepsilon \leq 1$, thus proving the lemma.

The case of connected components $I$ of type 2 of $\Omega^{c}$ is much more complicated. The difficulty is that if the generator of the stabilizer of $I$ is subexponentially distorted in $G$, then there exist exponentially many $(n, \varepsilon)$-separated points inside $I$, and hence a relevant part of the entropy is "concentrated" in $I$. To deal with this problem, for each connected component $I$ of type 2 of $\Omega^{c}$ we denote by $p_{I}$ its middle point, and then we define $\ell_{I}: G \rightarrow \mathbb{N}_{0}$ as follows. Let $h$ be the generator of the stabilizer of $I$ such that $h(x)>x$ for all $x$ in $I$. For each $f \in G$ the element $f h f^{-1}$ is the generator of the stabilizer of $f(I)$ with the analogous property. We then let $\ell_{I}(f)=|r|$, where $r$ is the unique integer such that

$$
f h^{r} f^{-1}\left(p_{f(I)}\right) \leq f\left(p_{I}\right)<f h^{r+1} f^{-1}\left(p_{f(I)}\right) .
$$

Lemma 3. For all $f, g$ in $G$ one has

$$
\ell_{I}(g \circ f) \leq \ell_{f(I)}(g)+\ell_{I}(f)+1 .
$$

Proof. Let $r$ be the unique integer such that

$$
\left(f h f^{-1}\right)^{r}\left(p_{f(I)}\right) \leq f\left(p_{I}\right)<\left(f h f^{-1}\right)^{r+1}\left(p_{f(I)}\right),
$$

and let $s$ be the unique integer for which

$$
\left(g f h f^{-1} g^{-1}\right)^{s}\left(p_{g f(I)}\right) \leq g\left(p_{f(I)}\right)<\left(g f h f^{-1} g^{-1}\right)^{s+1}\left(p_{g f(I)}\right),
$$

so that

$$
\ell_{I}(f)=|r|, \quad \ell_{f(I)}(g)=|s|
$$

We then have

$$
g^{-1}\left(g f h f^{-1} g^{-1}\right)^{s}\left(p_{g f(I)}\right) \leq p_{f(I)}<g^{-1}\left(g f h f^{-1} g^{-1}\right)^{s+1}\left(p_{g f(I)}\right),
$$

that is,

$$
\left(f h f^{-1}\right)^{s} g^{-1}\left(p_{g f(I)}\right) \leq p_{f(I)}<\left(f h f^{-1}\right)^{s+1} g^{-1}\left(p_{g f(I)}\right) .
$$


Therefore,

$\left(f h f^{-1}\right)^{r}\left(f h f^{-1}\right)^{s} g^{-1}\left(p_{g f(I)}\right) \leq f\left(p_{I}\right)<\left(f h f^{-1}\right)^{r+1}\left(f h f^{-1}\right)^{s+1} g^{-1}\left(p_{g f(I)}\right)$,

and hence

$$
\left(f h f^{-1}\right)^{r+s} g^{-1}\left(p_{g f(I)}\right) \leq f\left(p_{I}\right)<\left(f h f^{-1}\right)^{r+s+2} g^{-1}\left(p_{g f(I)}\right) .
$$

This easily gives

$$
g\left(f h f^{-1}\right)^{r+s} g^{-1}\left(p_{g f(I)}\right) \leq g f\left(p_{I}\right)<g\left(f h f^{-1}\right)^{r+s+2} g^{-1}\left(p_{g f(I)}\right),
$$

and thus

$$
\left(g f h f^{-1} g^{-1}\right)^{r+s}\left(p_{g f(I)}\right) \leq g f\left(p_{I}\right)<\left(g f h f^{-1} g^{-1}\right)^{r+s+2}\left(p_{g f(I)}\right) .
$$

This shows that $\ell_{I}(g f)$ equals either $|r+s|$ or $|r+s+1|$, which concludes the proof.

The following corollary is a direct consequence of the preceding lemma, but may be proved independently.

Corollary 1. For every $f \in G$ one has

$$
\left|\ell_{I}(f)-\ell_{f(I)}\left(f^{-1}\right)\right| \leq 1 .
$$

Proof. From (2) one obtains

$$
h^{-(r+1)}\left(p_{I}\right)<f^{-1}\left(p_{f(I)}\right) \leq h^{-r}\left(p_{I}\right)<h^{-r+1}\left(p_{I}\right),
$$

and hence $\ell_{f(I)}\left(f^{-1}\right)$ equals either $|r|$ or $|r+1|$. Since $\ell_{I}(f)=|r|$, the corollary follows.

4. The proof in the smooth case. To rule out the possibility of "concentration" of the entropy on a type 2 connected component $I$ of $\Omega^{c}$, in the $C^{2}$ case we will use classical control of distortion arguments in order to construct, starting from the function $\ell_{I}$, a kind of quasi-morphism from $G$ into $\mathbb{N}_{0}$. Slightly more generally, let $\mathcal{F}$ be any finite family of connected components of type 2 of $\Omega^{c}$. We denote by $\mathcal{F}^{G}$ the family of all intervals contained in the orbits of the intervals in $\mathcal{F}$. For each $f \in G$ we then define

$$
\ell_{\mathcal{F}}(f)=\sup _{I \in \mathcal{F}^{G}} \ell_{I}(f) .
$$

A priori, the value of $\ell_{\mathcal{F}}$ could be infinite. We claim, however, that, for groups of $C^{2}$ diffeomorphisms, this value is necessarily finite for every element $f$.

Proposition 1. For every finite family $\mathcal{F}$ of type 2 connected components of $\Omega^{c}$, the value of $\ell_{\mathcal{F}}(f)$ is finite for each $f \in G$.

To prove this proposition, we will need to estimate the function $\ell_{I}(f)$ in terms of the distortion of $f$ on the interval $I$. 
LEMma 4. For each fixed type 2 connected component I of $\Omega^{c}$ and every $g \in G$, the value of $\ell_{I}(g)$ is bounded from above by a number $L(V)$ depending on $V=\operatorname{var}\left(\log \left(\left.g^{\prime}\right|_{I}\right)\right)$, the total variation of the logarithm of the derivative of the restriction of $g$ to $I$.

Proof. Write $I=] a, b[$ and $g(I)=] \bar{a}, \bar{b}[$. If $h$ is a generator for the stabilizer of $I$, then for every $f \in G$ the value of $\ell_{I}(f)$ corresponds (up to some constant \pm 1 ) to the number of fundamental domains for the dynamics of $f h f^{-1}$ on $f(I)$ between the points $p_{f(I)}$ and $f\left(p_{I}\right)$, which in turn corresponds to the number of fundamental domains for the dynamics of $h$ on $I$ between $f^{-1}\left(p_{f(I)}\right)$ and $p_{I}$. Therefore, we need to show that there exist $c<d$ in $] a, b\left[\right.$ depending on $V$ and such that $g^{-1}\left(p_{g(I)}\right)$ belongs to $[c, d]$. We will show that this happens for the values

$$
c=a+\frac{|I|}{2 e^{V}} \quad \text { and } \quad d=b-\frac{|I|}{2 e^{V}} .
$$

We will just check that the first choice works, leaving the second one to the reader. By the Mean Value Theorem, there exist $x \in g(I)$ and $y \in\left[\bar{a}, p_{g(I)}\right]$ such that

$$
\left(g^{-1}\right)^{\prime}(x)=\frac{|I|}{|g(I)|}
$$

and

$$
\left(g^{-1}\right)^{\prime}(y)=\frac{\left|g^{-1}\left(\left[\bar{a}, p_{f(I)}\right]\right)\right|}{\left|\left[\bar{a}, p_{g(I)}\right]\right|}=\frac{g^{-1}\left(p_{g(I)}\right)-a}{|g(I)| / 2} .
$$

By the definition of the constant $V$, we have $\left(g^{-1}\right)^{\prime}(x) /\left(g^{-1}\right)^{\prime}(y) \leq e^{V}$. This gives

$$
e^{V} \geq \frac{|I| /|g(I)|}{2\left(g^{-1}\left(p_{g(I)}\right)-a\right) /|g(I)|}=\frac{|I|}{2\left(g^{-1}\left(p_{g(I)}\right)-a\right)},
$$

thus proving that $g^{-1}\left(p_{g(I)}\right) \geq a+|I| / 2 e^{V}$, as we wanted to show.

Proof of Proposition 1. Let $J=] \bar{a}, \bar{b}[$ be an interval in the $G$-orbit of $I=] a, b\left[\right.$. If $g=g_{i_{n}} \cdots g_{i_{1}}, g_{i_{j}} \in \Gamma$, is an element of minimal length sending $I$ to $J$, then the intervals $I, g_{i_{1}}(I), g_{i_{2}} g_{i_{1}}(I), \ldots, g_{i_{n-1}} \cdots g_{i_{2}} g_{i_{1}}(I)$ have pairwise disjoint interiors. Therefore,

$$
\operatorname{var}\left(\log \left(\left.g^{\prime}\right|_{I}\right)\right) \leq \sum_{j=0}^{n-1} \operatorname{var}\left(\log \left(\left.g_{i_{j+1}}^{\prime}\right|_{g_{i_{j}} \cdots g_{i_{1}}(I)}\right) \leq \sum_{h \in \Gamma} \operatorname{var}\left(\log \left(h^{\prime}\right)\right)=: W .\right.
$$

Moreover, setting $V=\operatorname{var}\left(\log \left(f^{\prime}\right)\right)$, we have

$$
\operatorname{var}\left(\log \left(\left.(f g)^{\prime}\right|_{I}\right)\right) \leq \operatorname{var}\left(\log \left(\left.g^{\prime}\right|_{I}\right)\right)+\operatorname{var}\left(\log \left(f^{\prime}\right)\right)=W+V .
$$


By Lemmas 3 and 4 and Corollary 1,

$$
\begin{aligned}
\ell_{J}(f) & \leq \ell_{J}\left(g^{-1}\right)+\ell_{I}(f g)+1 \leq \ell_{I}(g)+\ell_{I}(f g)+2 \\
& \leq L(W)+L(W+V)+2 .
\end{aligned}
$$

This proves the assertion of the proposition when $\mathcal{F}$ consists of a single interval. The case of general finite $\mathcal{F}$ follows easily.

For a given $\varepsilon>0$ we define $\ell_{\varepsilon}=\ell_{\mathcal{F}_{\varepsilon}}$, where $\mathcal{F}_{\varepsilon}=\left\{I_{1}, \ldots, I_{k}\right\}$ is the family of all connected components of $\Omega^{c}$ having length greater than or equal to $\varepsilon$, with $k=k(\varepsilon)$. Notice that, by Lemma 3, for every $f, g$ in $\Gamma$ one has

$$
\ell_{\varepsilon}(g f) \leq \ell_{\varepsilon}(g)+\ell_{\varepsilon}(f)+1 .
$$

Lemma 5. There exist constants $A(\varepsilon)>0$ and $B(\varepsilon)$ with the following property: If $x_{1}, \ldots, x_{m}$ are points in a single connected component of type 2 of $\Omega^{c}$ and $x_{i}, x_{j}$ are $(\varepsilon, n)$-separated for every $i \neq j$, then $m \leq A(\varepsilon) n+B(\varepsilon)$.

Proof. Write $c_{\varepsilon}=\max \left\{\ell_{\varepsilon}(g): g \in \Gamma\right\}$ (according to Proposition 1, the value of $c_{\varepsilon}$ is finite). Let $I$ be the type 2 connected component of $\Omega^{c}$ containing $x_{1}, \ldots, x_{m}$. We may assume that $x_{1}<\cdots<x_{m}$. For each $1 \leq$ $i \leq k$ let $h_{i}$ be the generator of $\operatorname{St}\left(I_{i}\right)$. Notice that $\ell_{\varepsilon}\left(h_{i}^{r}\right) \geq|r|$ for all $r \in \mathbb{Z}$.

If $f$ is an element in $B_{\Gamma}(n)$ sending $I$ to some $I_{i}$, then the number of points which are $\varepsilon$-separated by $f$ is less than or equal to $1 / \varepsilon+1$. We claim that the number of elements in $B_{\Gamma}(n)$ sending $I$ to $I_{i}$ is bounded above by $4 n c_{\varepsilon}+4 n-1$. Indeed, if $g$ also sends $I$ onto $I_{i}$ then $g f^{-1} \in \operatorname{St}\left(I_{i}\right)$, hence $g f^{-1}=h_{i}^{r}$ some $r$. Therefore, using (3) one obtains $|r| \leq \ell_{\varepsilon}\left(h_{i}^{r}\right) \leq$ $2 n c_{\varepsilon}+2 n-1$.

Since the previous arguments apply to each type 2 interval $I_{i}$, we have

$$
m \leq k(1 / \varepsilon+1)\left(4 n c_{\varepsilon}+4 n-1\right) .
$$

Therefore, letting

$$
A(\varepsilon)=(4 k+4 k / \varepsilon)\left(1+c_{\varepsilon}\right) \text { and } B(\varepsilon)=-(k+k / \varepsilon)
$$

concludes the proof.

To conclude the proof of Theorem A, the following notation will be useful.

Notation 1 . Given $\varepsilon>0$ and $n \in \mathbb{N}$, we denote by $s(n, \varepsilon)$ the largest cardinality of an $(n, \varepsilon)$-separated subset of $\mathrm{S}^{1}$. Likewise, $s_{\Omega}(n, \varepsilon)$ will denote the largest cardinality of an $(n, \varepsilon)$-separated set contained in the nonwandering set.

Proof of Theorem A. Fix $0<\varepsilon<1 / 2 L$, where $L$ is a common Lipschitz constant for the elements in $\Gamma$. We will show that, for some function $p_{\varepsilon}$ growing linearly in $n$ (and whose coefficients depend on $\varepsilon$ ), one has

$$
s(n, \varepsilon) \leq p_{\varepsilon}(n) s_{\Omega}(n, \varepsilon)+p_{\varepsilon}(n) .
$$


Actually, any function $p_{\varepsilon}$ with subexponential growth and satisfying such an inequality suffices. Indeed, taking the logarithm of both sides, dividing by $n$, and passing to the limit implies that

$$
h_{\Gamma}\left(G \circlearrowright \mathrm{S}^{1}, \varepsilon\right)=h_{\Gamma}(G \circlearrowright \Omega, \varepsilon) .
$$

Letting $\varepsilon$ go to zero gives

$$
h_{\Gamma}\left(G \circlearrowright \mathrm{S}^{1}\right) \leq h_{\Gamma}(G \circlearrowright \Omega) .
$$

Since the opposite inequality is obvious, this shows the desired equality between the entropies.

To show (4), fix an $(n, \varepsilon)$-separated set $S$ containing $s(n, \varepsilon)$ points. Let $n_{\Omega}$ (resp. $n_{\Omega^{c}}$ ) be the number of points in $S$ which are in $\Omega$ (resp. in $\Omega^{c}$ ). Obviously, $s(n, \varepsilon)=n_{\Omega}+n_{\Omega^{c}}$. Let $t=t_{S}$ be the number of connected components of $\Omega^{c}$ containing points in $S$, and let $l=[t / 2]$, where [.] denotes integer part. We will show that there exists an $(n, \varepsilon)$-separated set $T$ contained in $\Omega$ and having cardinality $l$. This will obviously give $s_{\Omega}(n, \varepsilon) \geq l$. The inequalities $t \leq 2 l+1$ and $n_{\Omega} \leq s_{\Omega}(n, \varepsilon)$, together with Lemmas 2 and 3 , will imply that

$$
\begin{aligned}
s(n, \varepsilon) & =n_{\Omega}+n_{\Omega^{c}} \leq n_{\Omega}+t k(1+1 / \varepsilon)\left(4 n c_{\varepsilon}+4 n-1\right) \\
& \leq s_{\Omega}(n, \varepsilon)+\left(2 s_{\Omega}(n, \varepsilon)+1\right) k(1+1 / \varepsilon)\left(4 n c_{\varepsilon}+4 n-1\right),
\end{aligned}
$$

thus showing (4).

To show the existence of the set $T$ with the properties above, we proceed in a constructive way. Let us enumerate the connected components of $\Omega^{c}$ containing points in $S$ in a cyclic way as $I_{1}, \ldots, I_{t}$. Now for each $1 \leq i \leq l$ choose a point $t_{i} \in \Omega$ between $I_{2 i-1}$ and $I_{2 i}$, and let $T=\left\{t_{1}, \ldots, t_{l}\right\}$. We need to check that, for $i \neq j$, the points $t_{i}$ and $t_{j}$ are $(n, \varepsilon)$-separated. Now by construction, for each $i \neq j$ there exist at least two different points $x, y$ in $S$ contained in the interval of smallest length in $S^{1}$ joining $t_{i}$ and $t_{j}$. Since $S$ is $(n, \varepsilon)$-separated, there exist $m \leq n$ and $g_{i_{1}}, \ldots, g_{i_{m}}$ in $\Gamma$ such that $\operatorname{dist}(h(x), h(y)) \geq \varepsilon$, where $h=g_{i_{m}} \cdots g_{i_{2}} g_{i_{1}}$. Unfortunately, because of the topology of the circle, this does not imply that $\operatorname{dist}\left(h\left(t_{i}\right), h\left(t_{j}\right)\right) \geq \varepsilon$. However, the proof will be finished if we show that

$$
\operatorname{dist}\left(g_{i_{r}} \cdots g_{i_{1}}\left(t_{i}\right), g_{i_{r}} \cdots g_{i_{1}}\left(t_{j}\right)\right) \geq \varepsilon \quad \text { for some } 0 \leq r \leq m .
$$

This claim is obvious if $\operatorname{dist}\left(t_{i}, t_{j}\right) \geq \varepsilon$. If this is not the case then, by the definition of the constants $\varepsilon$ and $L$, the length of the interval $\left[g_{i_{1}}\left(t_{i}\right), g_{i_{1}}\left(t_{j}\right)\right]$ is smaller than $1 / 2$, and hence it coincides with the distance between its endpoints. If this distance is at least $\varepsilon$, then we are done. If not, the same argument shows that the length of the interval $\left[g_{i_{2}} g_{i_{1}}\left(t_{i}\right), g_{i_{2}} g_{i_{1}}\left(t_{j}\right)\right]$ is smaller than $1 / 2$ and coincides with the distance between its endpoints. If this length is at least $\varepsilon$, then we are done. If not, we continue the procedure. Clearly, there must be some integer $r \leq m$ such that the length of the 
interval $\left[g_{i_{r-1}} \cdots g_{i_{1}}\left(t_{i}\right), g_{i_{r-1}} \cdots g_{i_{1}}\left(t_{j}\right)\right]$ is smaller than $\varepsilon$, and the one of $\left[g_{i_{r}} \cdots g_{i_{1}}\left(t_{i}\right), g_{i_{r}} \cdots g_{i_{1}}\left(t_{j}\right)\right]$ is greater than or equal to $\varepsilon$. As before, the length of the next interval will be forced to be smaller than $1 / 2$, and hence it will coincide with the distance between its endpoints. This shows (5) and concludes the proof of Theorem A.

\section{The proof in the absence of subexponentially distorted ele-} ments. Recall that topological entropy is invariant under topological conjugacy. Therefore, due to [3, Théorème $\mathrm{D}]$, in order to prove Theorem $\mathrm{B}$ we may assume that $G$ is a group of bi-Lipschitz homeomorphisms. Let $L$ be a common Lipschitz constant for the elements in $\Gamma$. Fix again $0<\varepsilon<1 / 2 L$, and let $I_{1}, \ldots, I_{k}$ be the connected components of $\Omega^{c}$ having length greater than or equal to $\varepsilon$. Let $h_{i}$ be a generator for the stabilizer of $I_{i}$ (with $h_{i}=\mathrm{Id}$ in case $I_{i}$ is of type 1). Consider the minimal non-decreasing function $q_{\varepsilon}$ such that, for each of the non-trivial $h_{i}$ 's, one has $q_{\varepsilon}\left(\left\|h_{i}^{r}\right\|\right) \geq r$ for all positive $r$. We will show that (4) holds for the function

$$
p_{\varepsilon}(n)=2 k(1+1 / \varepsilon)\left(2 q_{\varepsilon}(2 n)+1\right)+1 .
$$

Notice that, by assumption, this function $p_{\varepsilon}$ grows at most subexponentially in $n$. Hence, as in the case of Theorem A, inequality (4) allows us to finish the proof of the equality between the entropies.

The main difficulty in showing (4) in this case is that Lemma 5 is no longer available. However, the following still holds.

Lemma 6. If $x_{1}, \ldots, x_{m}$ are points in a single type 2 connected component $I$ of $\Omega^{c}$ having length at least $\varepsilon$, and $x_{i}, x_{j}$ are $(\varepsilon, n)$-separated for all $i \neq j$, then $m \leq k(1 / \varepsilon+1)\left(2 q_{\varepsilon}(2 n)+1\right)$.

Proof. Let $I$ be the type 2 connected component of $\Omega^{c}$ containing $x_{1}, \ldots, x_{m}$. We may assume that $x_{1}<\cdots<x_{m}$. If $f$ is an element in $B_{\Gamma}(n)$ sending $I$ to some $I_{i}$, then the number of points which are $\varepsilon$-separated by $f$ is less than or equal to $1 / \varepsilon+1$. We claim that the number of elements in $B_{\Gamma}(n)$ sending $I$ to $I_{i}$ is bounded above by $q_{\varepsilon}(r)$. Indeed, if $g$ also sends $I$ to $I_{i}$ then $g f^{-1} \in \operatorname{St}\left(I_{i}\right)$, hence $g f^{-1}=h_{i}^{r}$ some $r$. Therefore,

and hence

$$
2 n \geq\left\|g f^{-1}\right\|=\left\|h_{i}^{r}\right\|,
$$

$$
q_{\varepsilon}(2 n) \geq q_{\varepsilon}\left(\left\|h_{i}^{r}\right\|\right) \geq|r| .
$$

Since the previous arguments apply to each type 2 interval $I_{i}$, this gives

$$
m \leq k(1 / \varepsilon+1)\left(2 q_{\varepsilon}(2 n)+1\right),
$$

thus proving the lemma.

To show (4) in the present case, we proceed as in the proof of Theorem A. We fix an $(n, \varepsilon)$-separated set $S$ containing $s(n, \varepsilon)$ points. We let $n_{\Omega}$ 
(resp. $n_{\Omega^{c}}$ ) be the number of points in $S$ which are in $\Omega$ (resp. in $\Omega^{c}$ ), so that $s(n, \varepsilon)=n_{\Omega}+n_{\Omega^{c}}$. Let $t=t_{S}$ be the number of connected components of $\Omega^{c}$ containing points in $S$, and let $l=[t / 2]$. As before, one can show that there exists an $(n, \varepsilon)$-separated set contained in $\Omega$ and having cardinality $l$. This will obviously give $s_{\Omega}(n, \varepsilon) \geq l$. The inequalities $t \leq 2 l+1$ and $n_{\Omega} \leq s_{\Omega}(n, \varepsilon)$ still hold. Using Lemmas 2 and 6 one now obtains

$$
\begin{aligned}
s(n, \varepsilon) & =n_{\Omega}+n_{\Omega^{c}} \leq n_{\Omega}+t k(1+1 / \varepsilon)\left(2 q_{\varepsilon}(2 n)+1\right) \\
& \leq s_{\Omega}(n, \varepsilon)+\left(2 s_{\Omega}(n, \varepsilon)+1\right) k(1+1 / \varepsilon)\left(2 q_{\varepsilon}(2 n)+1\right) .
\end{aligned}
$$

This concludes the proof of Theorem B.

Acknowledgments. I would like to thank Andrés Navas for introducing me to this subject and his continuous support during this work, which was partially funded by Research Network on Low Dimensional Dynamical Systems (PBCT-Conicyt's project ADI 17). I would also extend my gratitude to both the referee and the editor for pointing out a subtle error in the original version of this paper.

\section{References}

[1] D. Calegari and M. Freedman, Distortion in transformation groups, Geom. Topology 10 (2006), 267-293.

[2] J. Cantwell and L. Conlon, Poincaré-Bendixson theory for leaves of codimension one, Trans. Amer. Math. Soc. 265 (1981), 181-209.

[3] B. Deroin, V. Kleptsyn et A. Navas, Sur la dynamique unidimensionnelle en régularité intermédiaire, Acta Math. 199 (2007), 199-262.

[4] E. Ghys, Groups acting on the circle, Enseign. Math. 47 (2001), 329-407.

[5] E. Ghys, R. Langevin et P. Walczak, Entropie géométrique des feuilletages, Acta Math. 160 (1988), 105-142.

[6] G. Hector, Architecture des feuilletages de classe $C^{2}$, Astérisque 107-108 (1983), $243-258$.

[7] A. Navas, Groups of Circle Diffeomorphisms, forthcoming book; Spanish version: Ensaios Matemáticos 13, Brazil. Math. Soc., 2007.

[8] - Growth of groups and diffeomorphisms of the circle, Geom. Funct. Anal. 18 (2008), 988-1028.

[9] P. Walczak, Dynamics of Foliations, Groups and Pseudogroups, IMPAN Monogr. Math. 64, Birkhäuser, Basel, 2004.

Departamento de Matemáticas

Facultad de Ciencias

Universidad de Chile

Las Palmeras 3425, Ñuñoa

Santiago, Chile

E-mail: ejorquer@u.uchile.cl

Received 15 September 2008;

in revised form 25 February 2009 